\title{
QUANTIFICAÇÃO DO DESMATAMENTO NA RESERVA FLORESTAL DE MECUBURI - MOÇAMBIQUE
}

\author{
Sosdito Estevão Mananze ${ }^{1}$ \\ João Neves Silva ${ }^{2}$ \\ Maria Jose Perestrelo Vasconcelos ${ }^{3}$
}

\section{Resumo}

Os desmatamentos nos países em desenvolvimento contribuem com 20 a $25 \%$ das emissões globais de dióxido de carbono. O presente estudo teve como objetivo determinar as taxas de desmatamento na reserva florestal de Mecuburi. Com recurso ao software ENVI 4.5, classificaram-se imagens do satélite Landsat de três datas (2002, 2007 e 2011), estimando-se em seguida as taxas de desmatamento entre os anos em estudo. Posteriormente, através da aplicação do modelo GEOMOD do software Idrisi Taiga, produziu-se uma previsão da localização do desmatamento para o ano 2020. No período em análise, a área florestal sofreu uma redução a uma taxa anual bruta de desmatamento de 2165 ha/ano. O desmatamento projetado para 2020 incide na zona norte da reserva. Um projeto REDD na reserva contribuiria para reduzir a desmatamento.

Palavras-chaves: Desmatamento; Mecuburi; sensoriamento remoto; alteração de cobertura/uso de solo.

\section{QUANTIFICATION OF DEFORESTATION IN THE MECUBURI FOREST RESERVE - MOZAMBIQUE}

\begin{abstract}
Deforestation in developing countries accounts for 20 to $25 \%$ of the global carbon emissions. This study intended to estimate the rates of deforestation within Mecuburi forest reserve. ENVI 4.5 software was used to map the land cover/use changes between three dates, 2002, 2007 and 2011. Gross and net deforestation rates were calculated and the location of deforestation in 2020 was projected using GEOMOD, an extension of IDRISI TAIGA software. Within the analyzed time interval, the forest area was reduced at a gross rate of $2165 \mathrm{ha} /$ year. Most of the projected deforestation is located to the north of the reserve. A REDD project could contribute to reduce deforestation within the reserve.

\footnotetext{
${ }^{1}$ Graduado em Engenharia Florestal, Mestre em Gestão e Conservação de Recursos Naturais. Atualmente é Docente Assistente na Escola Superior de Desenvolvimento Rural da Universidade Eduardo Mondlane. Email: blessestevao@gmail.com

${ }^{2}$ Licenciado e doutorado em Engenharia Florestal, tendo um mestrado em Matemática Aplicada. É atualmente investigador do CE3C-CCIAM (http://ce3c.ciencias.ulisboa.pt/teams/user/?id=24) onde está a trabalhar na adaptação às mudanças climáticas a nível local. Email: joaonevessilva@gmail.com

${ }^{3}$ Doutora em gestão de recursos naturais renováveis pela Universidade do Arizona, tendo um mestrado em gestão de bacias hidrográficas e uma licenciatura em Silvicultura. É atualmente investigadora no Instituto Superior de Agronomia (www.isa.ulisboa.pt) onde está a trabalhar no projeto ODYSSEA ( http://lgkyd7.xara.hosting/odyssea) e no desenvolvimento de um sistema para Zonamento Agroecológico em África Ocidental no âmbito do projeto EU-ACTIVA. Email: maria.perestrelo@gmail.com
} 
Keywords: Deforestation; Mecuburi; remote sensing; land cover/use change.

\section{CUANTIFICACIÓN DE LA DEFORESTACIÓN EN RESERVA FORESTAL MECUBURI - MOZAMBIQUE}

\section{Resumen}

La deforestación en los países en desarrollo contribuye en un 20 a 25\% de las emisiones globales de dióxido de carbono. Este estudio tuvo como objetivo determinar la tasa de deforestación en la reserva forestal Mecuburi. Con uso de software ENVI 4.5, las imágenes de satélite Landsat se clasificaron tres fechas (2002, 2007 y 2011), a continuación, la estimación de las tasas de deforestación entre los años estudiados. Posteriormente, mediante la aplicación del modelo GEOMOD software Idrisi Taiga, se a producido una previsión de ubicación de la deforestación para el año 2020. En este período, la superficie forestal se redujo a una tasa anual de deforestación bruta de 2.165 ha / año. La deforestación proyectada para el año 2020 se centra en la parte norte de la reserva. Un proyecto REDD en la reserva ayudaría a reducir la deforestación.

Palabras clave: La deforestación; Mecuburi; detección remota; cambiar cubierta / uso del suelo.

\section{INTRODUÇÃO}

As florestas tropicais são muito ricas em biodiversidade e desempenham várias funções no sequestro de carbono, produção primária líquida e no ciclo hidrológico (Daily et al., 2000; Houghton et al., 2000; DeFries et al., 1999). Nas últimas décadas, o desmatamento tornou-se a principal causa de alteração da cobertura e uso do solo nos trópicos, causando vulnerabilidade às populações humanas locais (Cincotta \& Engelman, 2000; Angelsen \& Kaimowitz, 1999; Achard et al., 1998; Barrow, 1991).

Estima-se que cerca de 20 a $25 \%$ das emissões de dióxido de carbono resultem do desmatamento e degradação florestal nos países em vias de desenvolvimento (Harris et al., 2008; Houghton et al., 2000). Alguns autores indicam o crescimento populacional, a pobreza, a extração da madeira e agricultura itinerante como os principais fatores do desmatamento nos trópicos (Sedano \& Ferrão, 2005; Mather \& Needle, 2000; Allen \& Barnes, 1985). Outros porém, defendem que estes fatores só contribuem para o aumento do desmatamento na presença de fatores políticos, sociais e infraestruturas que influenciem a alteração de oportunidades económicas (Geist \& Lambin, 2001; Angelsen \& Kaimowitz, 1999; Hecht, 1985). Por exemplo, a fraca fiscalização florestal em muitos países africanos contribui para o 
aumento da extracção ilegal da madeira e dos impactos ambientais associados (Martens et al., 2000).

O desmatamento evitado foi reconhecido como elegível para a compensação por redução de emissões de dióxido de carbono. Como resultado, foi lançado o mecanismo para a Redução de Emissões por Desmatamento e Degradação Florestal (REDD) nos acordos pósQuioto no âmbito da United Nations Framework Convention on Climate Change (UNFCCC). À luz deste mecanismo, países em desenvolvimento podem receber incentivos financeiros para reduzir as emissões por desmatamento e degradação florestal, através de uma gestão sustentável das florestas (UNFCCC, 2006). Há consenso de que o REDD é uma oportunidade para estabelecer parcerias entre países desenvolvidos e em desenvolvimento em prol da estabilidade do clima global e de um desenvolvimento sustentável. A redução do desmatamento e degradação florestal em conjunto com acções de florestação e reflorestamento, associadas a uma gestão sustentável das florestas podem reduzir substancialmente os custos para alcançar as metas de estabilização climática e, ao mesmo tempo, contribuir para a melhoria das condições de vida das comunidades locais (Rose et al., 2007).

Dados do Inventário Florestal Nacional (Marzoli, 2008), indicam que a cobertura florestal natural em Moçambique é cerca de 40,1 milhões de hectares, dos quais 26,9 milhões constituem a floresta produtiva e 13 milhões estão integrados em áreas de conservação. Segundo o mesmo autor, a taxa anual de desmatamento em Moçambique, entre 1990 e 2002 foi de $0,58 \%$, o que representa uma conversão anual de 219000 ha de florestas para outros tipos de uso. Esta taxa foi estimada com base num modelo matemático similar ao adotado pela FAO (FRA, 1993), o qual estabelece uma correlação entre a pressão populacional e a cobertura florestal. As principais causas de desmatamento em Moçambique são a agricultura, a colheita de lenha e produção de carvão, e a expansão de zonas habitacionais (Marzoli, 2008; Argola, 2004; Saket, 1994).

Sendo assim, o mecanismo REDD constitui, para Moçambique, uma oportunidade para uma gestão sustentável das florestas que promove os serviços dos ecossistemas e a biodiversidade em geral e que, ao mesmo tempo, gera renda para as comunidades locais. Neste contexto, foi elaborada a estratégia nacional de REDD (MICOA, 2010), a qual representa uma etapa importante na concepção da Proposta de Preparação de Aptidão/Elegibilidade do país para se beneficiar de fundos do REDD. 
Na estratégia do MICOA (2010) são identificadas várias áreas florestais ao longo do país, consideradas potenciais para uma intervenção segundo o mecanismo REDD. Todavia, para que uma determinada área seja elegível no âmbito do REDD é necessário, entre outros aspectos, conhecer a linha de base do desmatamento. Segundo Huettner et al., (2009), a linha de base corresponde ao padrão de distribuição espacial e à quantidade do desmatamento em um período mínimo de dez anos antes do início da intervenção REDD. É usada para prever a quantidade e a localização do desmatamento que ocorreria se nenhuma medida de proteção fosse implementada. Por seu turno, esta previsão do desmatamento futuro é comparada com o resultado de ações concretas, implementadas no âmbito de REDD, de modo a quantificar e localizar o desmatamento evitado. Portanto, conhecer a taxa de desmatamento é crucial para medir o desempenho das atividades de REDD na redução das emissões e, consequentemente, negociar de forma sustentável as metas de redução de emissões por desmatamento.

O trabalho teve como principal objetivo determinar as taxas de desmatamento e projetar a sua localização e foi realizado com recurso a sensoriamento remoto. Esta ferramenta tem sido largamente aplicada na cartografia da cobertura e uso do solo, para estimar taxas de desmatamento, determinar os atributos biofísicos e as implicações socioeconómicas e ambientais das alterações da cobertura do solo em áreas extensas e de difícil acesso (Skole \& Tucker, 1994). O sensoriamento remoto constitui uma opção economicamente mais acessível para a medição das alterações da cobertura florestal, comparada com a realização de inventários florestais. Os inventários continuam sendo essenciais para a validação da cartografia por sensoriamento remoto mas são, neste caso, realizados com uma menor frequência. Outra vantagem tem a ver com a disponibilidade gratuita de imagens do satélite Landsat, de cobertura global e com boa resolução espacial (30 metros) e temporal (16 dias) (USGS, 2008).

\section{Descrição da Área de Estudo}

A Reserva Florestal de Mecuburi (RFM) é a maior em Moçambique. Localiza-se no distrito de Mecuburi, província de Nampula, no Norte de Moçambique, sendo delimitada pelas seguintes coordenadas geográficas: 37 L 537778.30 E, 8387911.55 S; 37 L 542478.11 E, 8404412.00 S; 37 L 555956.45 E, 8389366.29 S. Foi criada pela Portaria número 8459 Boletim Oficial no 29, I Série, de 22 de Julho de 1950, com o objectivo de impedir o avanço 
da agricultura e conservar o ecossistema ribeirinho garantindo a protecção da bacia hidrográfica do rio Mecuburi (Aguiar Macedo, 1968).

A RFM insere-se numa região de relevo que varia das planícies de aluvião nas proximidades dos cursos de água e nas zonas adjacentes, áreas de média altitude (300 m e 500 m), às zonas montanhosas de Imala, entre 500 e $1200 \mathrm{~m}$ de altitude. Predomina o clima quente, oceânico, seco (na zona norte da região) a chuvoso (no restante). Dados do Instituto Nacional de Meteorologia, Estação de Mecuburi (2001), indicam temperaturas médias anuais de $25^{\circ} \mathrm{C}$, sendo Janeiro e Fevereiro os meses mais quentes (com 26,6 ${ }^{\circ} \mathrm{C}$ e $25,8^{\circ} \mathrm{C}$, respectivamente) e Julho o mês mais frio e seco (com $21,2^{\circ} \mathrm{C}$ ) (Ribeiro et al., 2002). A precipitação média anual varia entre os $500 \mathrm{~mm}$ e os $2000 \mathrm{~mm}$ nas zonas mais chuvosas. A reserva é atravessada por vários rios, permanentes e sazonais, sendo o rio Mecuburi o mais importante.

Segundo Costa (1998), o Miombo é o principal tipo de vegetação da reserva. A palavra "Miombo" provém de várias línguas faladas no centro de África e denota a presença e dominância de uma ou mais espécies do género Brachystegia e Julbernardia, e da espécie Isoberlina angolensis (Fabaceae, familia Caesalpinioideae) na região. De resto, o Miombo é o tipo florestal mais abundante na África sub-sahariana, numa extensão de aproximadamente 270 milhões de hectares (Campbell, 1996; Frost, 1996; Celender, 1983). Estima-se que existam no Miombo cerca de 8500 espécies de plantas superiores, das quais mais de $54 \%$ são endêmicas (Rodgers et al., 1996). Ao longo do rio Mecuburi encontra-se floresta de galeria, com dominância de espécies como Spirostachys africana, Schinziophyton rautaneniie, Milletia stuhlmanni e ainda algumas pequenas árvores como Friesodielsia obovatae e várias espécies do género Strychnus (Ribeiro et al., 2002).

Estima-se que vivam na área da reserva cerca de 40 mil pessoas integradas em 12 mil agregados familiares e distribuídos por 15 regulados (Awasse \& Mushove, 2000). A agricultura itinerante é a principal atividade de subsistência. É praticada maioritariamente em regime de consociação de culturas e em áreas com menos de 1 hectare (MAE, 2005). A Figura 01 representa a localização geográfica da área de estudo. 
Figura 01: Localização da reserva florestal de Mecuburi.

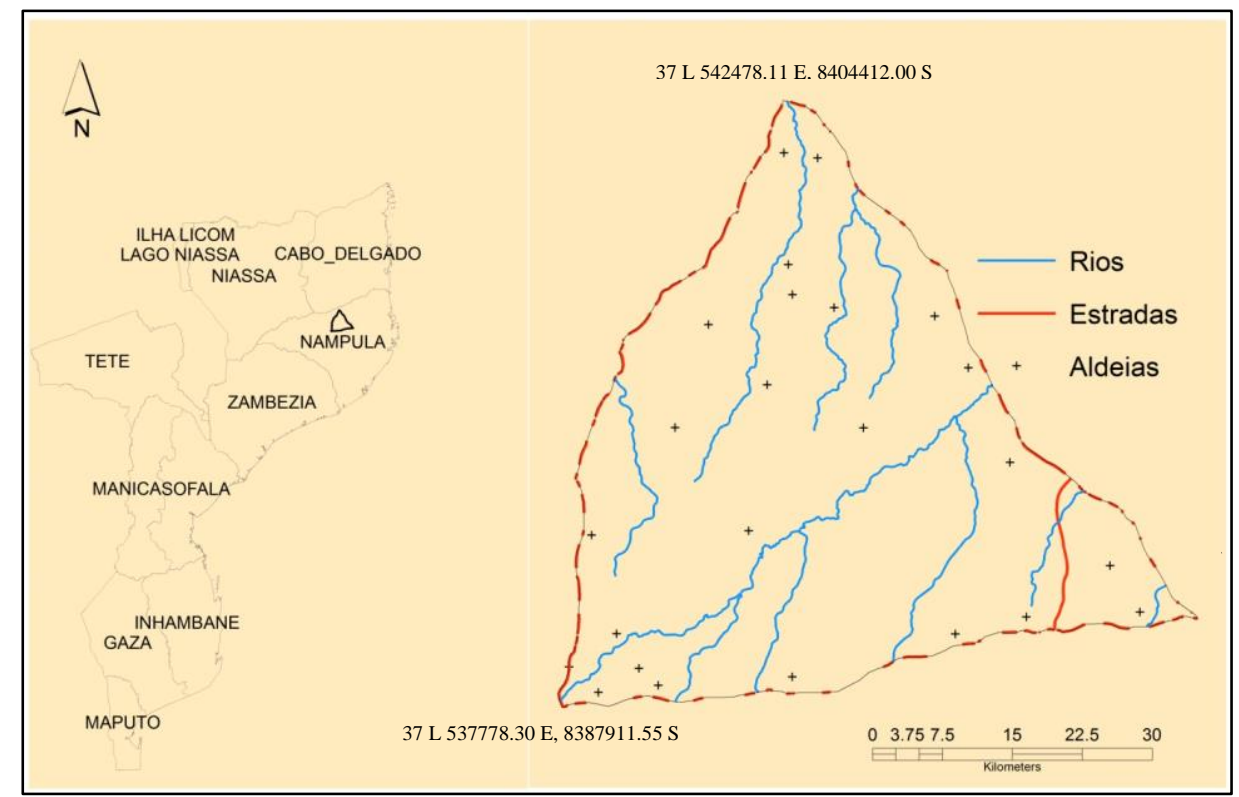

Fonte: http://www.cenacarta.com/modules.php?name=Downloads

\section{MÉTODOS}

\section{Aquisição de dados}

As imagens de satélite Landsat 7 ETM+ da área de estudo foram seleccionadas e baixadas gratuitamente a partir do sítio do USGS Global Visualization Viewer ${ }^{4}$. Todas as imagens estão geometricamente corrigidas. As imagens Landsat 7 ETM+ desta coleção têm uma resolução de 28,5 m e um erro de posicionamento inferior a $50 \mathrm{~m}$ (Tucker et al., 2004), sendo assim apropriadas para estudos de detecção de alterações da cobertura do solo.

A área de estudo é coberta pela imagem 165/70 (coluna/linha). Foram baixadas as imagens das seguintes datas: 14/07/2002, 11/06/2007/ e 06/06/2011. A seleção das imagens obedeceu aos seguintes critérios: Imagens do período seco; - permitem melhor distinção dos diferentes tipos de vegetação. O sinal verde corresponde à vegetação florestal, pois as gramíneas encontram-se secas por deficiência de água no solo; menor cobertura de nuvens as nuvens e as respectivas sombras projectadas obstruem a resposta espectral dos objetos à superfície; Sem áreas queimadas - provocam confusão com as respostas espectrais de outros

\footnotetext{
${ }^{4}$ http://glovis.usgs.gov/
} 
objetos e a subestimação da área de floresta, pois apesar de o fogo afectar mais o estrato herbáceo, o sinal da vegetação florestal fica alterado.

A informação geográfica sobre rios, estradas e aldeias dentro da área de estudo foi obtida a partir do sítio do Centro Nacional de Cartografia e Teledetecção de Moçambique (CENACARTA). ${ }^{5} \mathrm{O}$ modelo digital do terreno foi obtido a partir do USGS (2004) ${ }^{6}$.

\section{Pré-processamento}

Calibração Radiométrica Relativa

Tendo em conta que as imagens são de diversas datas, os efeitos atmosféricos e de ângulo de iluminação e observação são diferentes. A calibração radiométrica relativa é uma das técnicas usadas para normalizar as diferenças entre as imagens de diferentes datas, permitindo a sua comparação (Jensen et al., 2006; Tokola et al., 1998). Consiste em transformar as características espectrais das imagens com base na escala radiométrica de uma imagem considerada como de referência (Jensen et al., 2006). Neste estudo, tomou-se como base a imagem de Junho de 2007. Digitalizaram-se sobre as imagens, pequenas áreas de treino de objetos cuja reflectância deveria variar muito pouco devido às suas características. A variação observada entre cada par de imagens deve-se a variações das condições atmosféricas e de iluminação/observação. Na área de estudo estes objetos pseudo-invariantes consistem de afloramentos rochosos, bancos de areia e superfícies de água profunda. Através de equações de regressão linear estabeleceu-se uma relação entre as reflectâncias dos objetos em cada banda na imagem de referência de 11/06/2007 e nas imagens de 14/07/2002 e de 06/06/2011, e nas imagens de 29/07/2007, 05/07/2008; 24/07/2011 e 05/05/2010, estas últimas usadas para o preenchimento de lacunas.

Preenchimento de lacunas nas imagens

Em 31 de Maio de 2003, o sensor do satélite Landsat 7 Enhaced Thematic Mapper (ETM) registou uma avaria do Scan Line Corrector (SLC). Desde então, as imagens Landsat

\footnotetext{
${ }^{5} \mathrm{http}: / / \mathrm{www} \cdot c e n a c a r t a . c o m /$ modules.php?name=Downloads

${ }^{6}$ http://glcf.umiacs.umd.edu/data/srtm/
} 
ETM apresentam lacunas, resultando na perda de aproximadamente $22 \%$ dos dados (The Yale Center for Earth Observation, 2011).

Scaramuzza et al., (2004) desenvolveram uma técnica para preencher as lacunas de uma imagem (primária) com base em dados de outra imagem (secundária). Dado que não foi possível obter imagens completamente livres de nuvens, utilizou-se também a técnica de preenchimento de lacunas para eliminar as nuvens na área da reserva. Para o efeito, as nuvens e as respectivas sombras projectadas foram mascaradas (atribuição do valor zero), permitindo o seu preenchimento e obtenção de imagens livres de nuvens, pelo menos dentro dos limites da área de estudo (Figura 02).

As imagens secundárias, apresentadas na Tabela 01, foram seleccionadas com base nos seguintes critérios: com pouca cobertura de nuvens; com data o mais próximo possível das imagens primárias e/ou mesma estação do ano, permitindo assim minimizar o efeito das variações da fenologia da vegetação.

Tabela 01: Imagens usadas no preenchimento de lacunas.

\begin{tabular}{cccc}
\hline Linha/Coluna & 2007 & 2011 & Observação \\
\hline \multirow{3}{*}{$165 / 70$} & $11-07-2007$ & $06-06-2011$ & Primária \\
& $29-07-2007$ & $24-07-2011$ & Secundária \\
& $05-07-2008$ & $05-05-2010$ & Secundária \\
\hline
\end{tabular}

Fonte: Elaboração própria.

Figura 02: Parte de imagem Landsat da área de estudo: A - com lacunas resultantes da avaria do Scan Line Corrector (SLC), nuvens e respectivas sombras; B - com as lacunas, as nuvens e as sombras mascaradas; $\mathrm{C}$ - livre de lacunas, nuvens e sombras, como resultado do preenchimento com outras imagens.

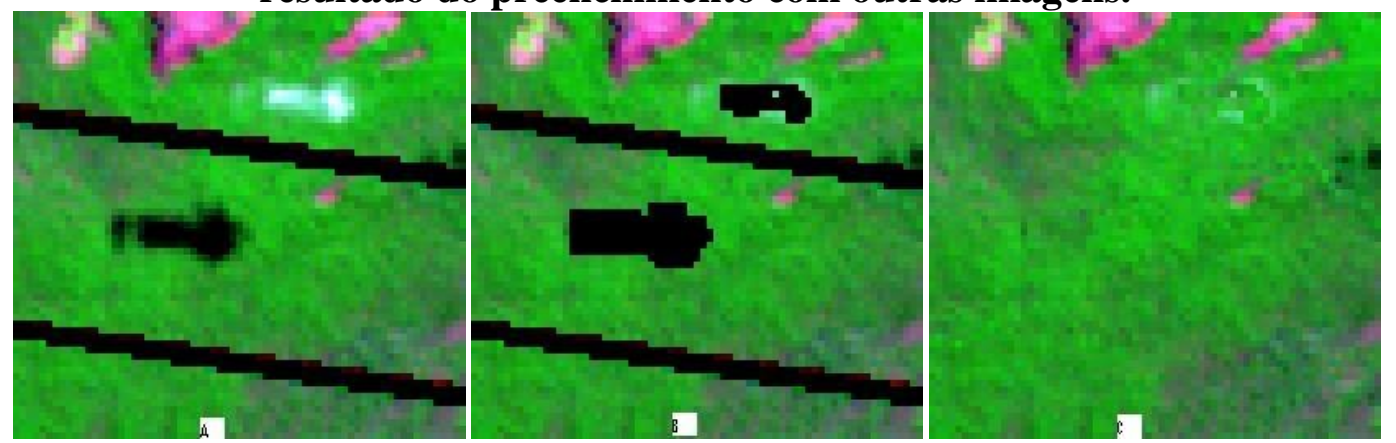

Fonte: Elaboração própria.

Classificação das Imagens Landsat

Sociedade e Território - Natal. Vol. 28, N. 1, p. 27-47. Jan./Jun. de 2016 
No presente estudo foi aplicado o classificador de Máxima Verossimilhança. É o classificador supervisionado mais usado e considerado um dos mais poderosos pois aplica a teoria de probabilidade Bayesiana (Perumal \& Bhaskaran, 2010; IDRISI Klimanjaro Guide 2004; Richards, 1995). Segundo Richards \& Jia (1999), todos os classificadores supervisionados obedecem aos seguintes passos: definição das classes de cobertura nos quais a imagem deverá ser classificada; captura e avaliação de áreas de treinamento para cada classe; atribuição de uma classe a cada pixel da imagem e avaliação da eficácia da classificação. Foram adotadas as seguintes categorias de cobertura/uso de solo:

I. Floresta densa: Inclui todas as áreas com vegetação lenhosa consistente com os padrões da definição de floresta em Moçambique (área com pelo menos 1 hectare de superfície com uma cobertura de copas igual ou superior a 10\%, com árvores de altura superior a 5 metros).

II. Floresta aberta: Áreas com cobertura de copas inferior a 10\% e com árvores de altura inferior a 5 metros. Inclui áreas arbustivas, arbustos em áreas húmidas e mosaicos de floresta com agricultura itinerante.

III. Agricultura: áreas com culturas agrícolas e ou agroflorestais cuja estrutura da vegetação está abaixo dos padrões de definição da floresta densa e floresta aberta.

IV. Água: áreas cobertas ou saturadas de água durante todo ano ou num determinado período.

V. Assentamentos humanos: inclui aldeias e infraestruturas de transporte.

VI. Afloramentos rochosos: áreas onde predominam os afloramentos rochosos.

VII. Solos agrícolas: Inclui áreas agrícolas sem culturas por estarem em período de colheita ou por terem sido recentemente preparadas para a sementeira.

VIII. Ardido: áreas ardidas.

A imagem composta (RGB) obtida com as bandas Landsat 7, 4 e 3 mostrou-se a mais conveniente para a extracção das áreas de treinamento. Estas consistem de amostras de pixéis com valores representativos de cada classe de interesse. Procurou-se sempre que possível atingir o valor de $100 \mathrm{~N}$ áreas de treinamento em cada classe, em que $\mathrm{N}$ é o número de bandas da imagem a classificar, valor aconselhado para alcançar uma classificação eficaz (Swain \& Davis, 1978).

Às 7 bandas do Landsat 7 ETM+ adicionou-se o índice NDVI - Normalized Difference Vegetation Index. O NDVI realça o sinal da vegetação verde, tendo em conta que 
ela absorve mais radiação na região do visível e reflete mais na do infravermelho. O NDVI é calculado com base na expressão: NDVI $=\frac{I V P-V}{I V P+V}$. No caso do Landsat com as bandas 3 (vermelho) e 4 (infravermelho próximo). Mais detalhes sobre o NDVI podem ser encontrados, por exemplo, em Holben et al., 1990.

Diferentes combinações de bandas foram usadas para avaliar a separabilidade entre as classes através da distância de Jeffries-Matusita (Richards \& Jia, 1999). A distância de Jeffries -Matusita (JM) calcula-se pela seguinte expressão: $J M=\sqrt{2\left(1-\mathrm{e}^{-\propto}\right)} ; \propto=$ $\frac{1}{8}\left(U_{u}-U_{b}\right)^{T} X\left[\frac{C_{u}+C_{b}}{2}\right]^{-1} X\left(U_{u}-U_{b}\right)+\left\{\frac{1}{2} \frac{\left(\cup_{u}+\cup_{b}\right)}{\sqrt{C_{u} X C_{b}}}\right\} ; u$ e $b$ são as classes em comparação; $\mathrm{C}_{u}$ e $\mathrm{C}_{\mathrm{b}}$ as matrizes de covariância das classes e $\mathrm{U}$ a média do vector da classe.

O cálculo desta medida permitiu melhorar a captura de áreas de treinamento, redefinindo o número de classes amostradas, agregando as semelhantes e/ou separando as diferentes. A distância de JM tem um limite máximo de 2, correspondente a classes completamente separáveis e um mínimo de 0 , denotando classes espectralmente idênticas.

\section{Cálculo das taxas de desmatamento}

As emissões de carbono por desmatamento e degradação florestal podem ser estimadas com base em taxas brutas ou taxas líquidas de alteração dos stocks. Em ambos os casos, o cálculo do desmatamento é feito com base na área de floresta no ano inicial (2002 no presente caso) do período de referência (banchmark map) e não na totalidade da área de estudo. No cálculo da taxa bruta assume-se que as árvores são removidas, a totalidade de carbono da sua biomassa é emitida e as áreas desmatadas permanecem como tal durante todo o período de referência. Na taxa líquida, pelo contrário, as áreas desmatadas que recuperem a cobertura florestal ao longo do período de referência são incluídas na classe floresta no ano final. No presente trabalho foram calculadas ambas as taxas. Para tal, os mapas de ocupação/uso do solo foram reclassificados, agregando as classes floresta densa e floresta aberta em uma só - classe floresta - e as restantes classes agregadas em outra - classe não floresta.

O cálculo foi efetuado utilizando uma extensão desenvolvida no programa ENVI IDL desmatamento, a qual permite determinar a quantidade e a localização de pixéis convertidos de floresta para não floresta e vice-versa, tendo como base a área florestal do início do período de referência. O número de pixéis é posteriormente convertido em área e a 
taxa de desmatamento calculada com base na equação de Puyravaud (2003): $R=\frac{1}{t 2-t 1}$ * $\ln \left[\frac{A 2}{A 1}\right]$, onde $\mathrm{A} 1$ é área florestal no início do período de referência $\left(\mathrm{t}_{1}\right)$ e $\mathrm{A}_{2}$ é área florestal no final do período de referência $\left(\mathrm{t}_{2}\right)$.

\section{Modelação espacial da localização do futuro desmatamento}

Modelos espaciais explícitos podem projetar a localização do desmatamento futuro com base em dados anteriores (Brown et al., 2006). Uma das razões chave para a utilização da modelação espacial no âmbito de REDD é que a cobertura/uso de solo projetado no futuro pode ser associado aos stocks de carbono florestal para estimar as emissões de $\mathrm{CO}_{2}$ correspondentes ao tipo de floresta e stock que estão naquela localização específica ao invés de se utilizarem valores médios conservadores (Harris et al., 2008). Neste trabalho, aplicou-se o modelo espacial GEOMOD do programa Idrisi Taiga (Hall et al., 1995; Pontius et al., 2001). É um modelo que simula o padrão espacial de alteração de cobertura/uso de solo para o futuro, mas também, para o passado. Tem sido aplicado com sucesso para a análise de linhas de base de desmatamento para projetos de sequestro de carbono (Harris et al., 2008; Brown et al., 2006). O GEOMOD foi concebido particularmente de modo a conferir uma máxima aplicação aos dados disponíveis nas regiões tropicais, quase sempre insuficientes e de baixa qualidade, daí a sua habilidade de fazer a calibração com base em apenas um mapa da cobertura/uso do solo (Pontius \& Chen, 2006; Pontius et al., 2001). Outros modelos espaciais requerem dados de pelo menos quatro anos para a calibração (Nualchawee et al., 1981; Gastellu-Etchegorry \& Sinulingga, 1988). No presente estudo usou-se informação de 2002 e 2011 para calibrar o modelo e depois simular a localização do desmatamento no período 2011 - 2020. Os seguintes dados foram usados para a modelação:

i. Mapa de cobertura do solo de 2002, com apenas duas classes - floresta e nãofloresta;

ii. Mapa do desmatamento bruto - consiste de um mapa com duas classes (florestalnão floresta), preparado com base no mapa das transições entre os três anos em análise (2002, 2007 e 2011). A classe floresta corresponde à área que se manteve como tal durante todo o período de referência enquanto a classe não floresta corresponde à combinação de todas as transições de floresta para não floresta; 
iii. Mapas dos fatores - fatores potencialmente correlacionados com o desmatamento na área de estudo. Foram considerados a topografia (elevação), as estradas, os rios e as aldeias. Os mapas criados consistem de distâncias euclidianas às estradas, aos rios e às aldeias e do modelo digital do terreno.

No processo de calibração, o modelo cria empiricamente um mapa de vulnerabilidade ao desmatamento, com base nos mapas dos fatores, no mapa da cobertura do solo de 2002 e na quantidade de desmatamento bruto entre 2002 e 2011. Parte-se do pressuposto segundo o qual, o risco de desmatamento diminui com o aumento da distância aos fatores de acessibilidade (estradas, rios e aldeias) e com o aumento da elevação do terreno. Fez-se uma combinação dos fatores considerando que todos os fatores têm igual peso na determinação do risco de desmatamento.

O mapa de vulnerabilidade apresenta valores elevados onde os mapas dos fatores têm características similares às zonas desmatadas no mapa de cobertura/uso de solo do ano inicial e, valores baixos onde os mapas dos fatores têm características similares às zonas não desmatadas no mapa de cobertura/uso de solo do ano inicial. Este mapa foi reclassificado resultando no mapa de potencial de alteração do cobertura/uso do solo. Na calibração, o GEOMOD produziu também um mapa simulado do desmatamento em 2011 selecionando pixéis com maior valor de vulnerabilidade até atingir a quantidade de desmatamento registada em 2011. De modo a aferir o poder preditivo do modelo, o mapa simulado foi comparado com o mapa do desmatamento bruto de referência. Esta comparação produz uma estatística (Kappa for location) que mede o grau de concordância do mapa de desmatamento simulado com o mapa de referência, quanto à localização do desmatamento (Pontius, 2002). O mapa de vulnerabilidade foi posteriormente usado para simular a localização do desmatamento no período 2011 - 2020 com base no mapa de desmatamento de 2011 e da taxa de desmatamento bruto extrapolada para o ano 2020.

\section{RESULTADOS}

\section{Taxa de desmatamento na reserva de Mecuburi}


Os mapas da Figura 03 indicam a localização do desmatamento bruto nos períodos 2002 - 2007 e 2002 - 2011 e o Quadro 01 apresenta as taxas de desmatamento líquido e bruto para os sub períodos 2002 - 2007 e 2007 - 2011 e para o período 2002 - 2011.

Figura 03: Mapas de desmatamento bruto nos períodos: A - 2002/2007; B - 2002/2011.
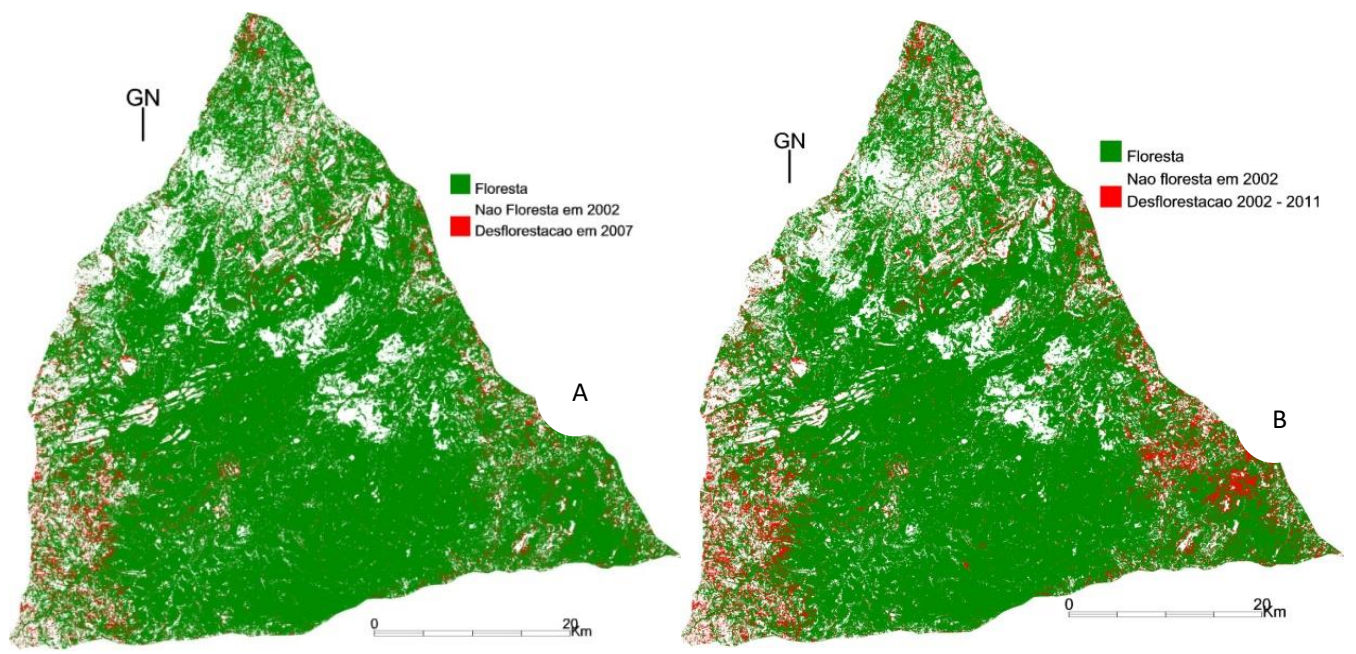

Fonte: Elaboração própria.

Os mapas mostram que o desmatamento localiza-se próximo das principais estradas que delimitam a reserva, sobretudo junto aos três vértices. Verifica-se também uma tendência de progressão do desmatamento em direção à parte central da reserva, onde se situa a maior porção da floresta densa.

Como era esperado, a taxa de desmatamento líquida é inferior à taxa bruta. Esta diferença reflete o fato de a taxa bruta captar a redução da área florestal do início do período de referência que, na taxa líquida é compensada pela inclusão das áreas regeneradas. A figura 04 apresenta a tendência da área florestal bruta e líquida ao longo do período de análise, calculada tendo em conta a área no início do período e as alterações ocorridas nos anos subsequentes.

Quadro 01: Desmatamento anual bruto e líquido (ha/ano) e respetivas taxas para os sub períodos 2002/2007, 2007/2011 e para o período de referência 2002/2011.

\begin{tabular}{|lllllll|}
\hline & \multicolumn{2}{c}{$2002 / 2007$} & \multicolumn{2}{c}{$2007 / 2011$} & \multicolumn{2}{c|}{ 2002/2011 } \\
\cline { 2 - 7 } Desmatamento & Área (ha/ano) & $\%$ & $\begin{array}{l}\text { Área } \\
\text { (ha/ano) }\end{array}$ & $\%$ & (ha/ano) & $\%$ \\
\hline Bruto & 2322,00 & 1,29 & 1969,85 & 1,16 & 2165,49 & 1,23 \\
Líquido & 641,47 & 0,35 & 1969,85 & 1,10 & 1231,86 & 0,72 \\
\hline
\end{tabular}

Fonte: Elaboração própria. 
Figura 04: Evolução da Área florestal bruta e líquida ao longo do período de referência.

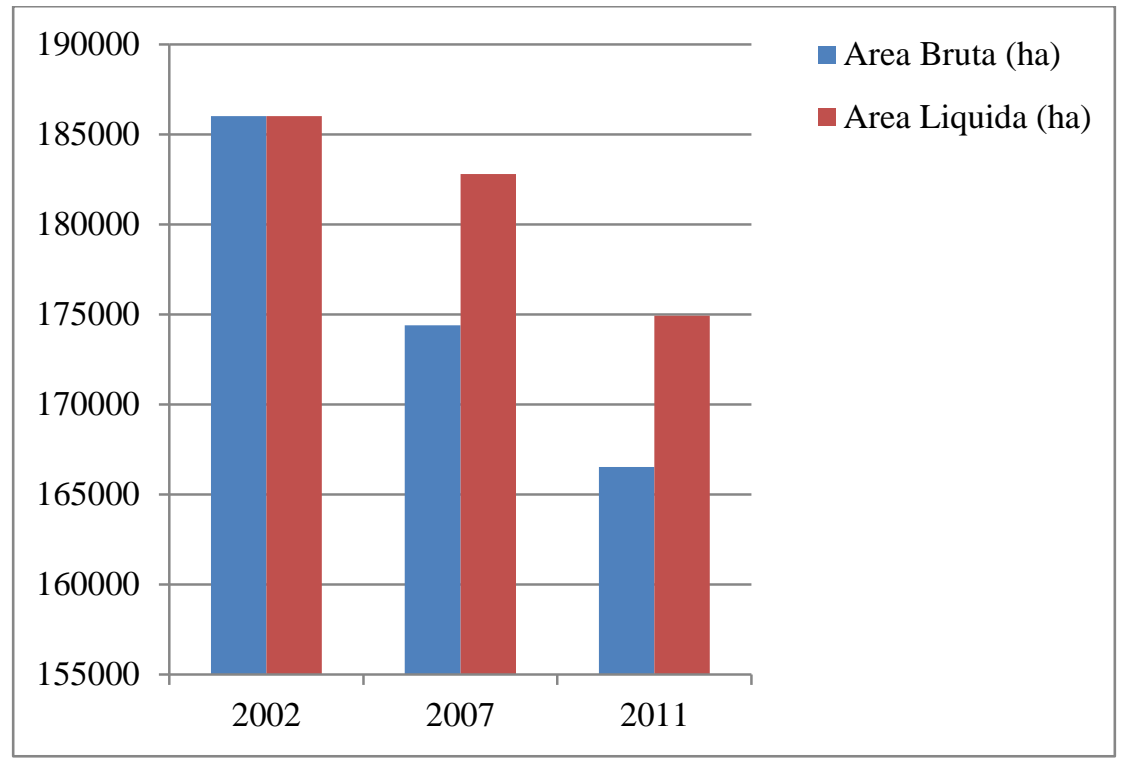

Fonte: Elaboração própria.

\section{Modelagem espacial do desmatamento}

A Figura 05 mostra o mapa de potencial de alteração da cobertura/uso do solo, resultado da reclassificação do mapa de vulnerabilidade ao desmatamento. A calibração resultou num Kappa for location de 0,74.

Figura 05: Mapa de potencial de alteração da cobertura/uso do solo.

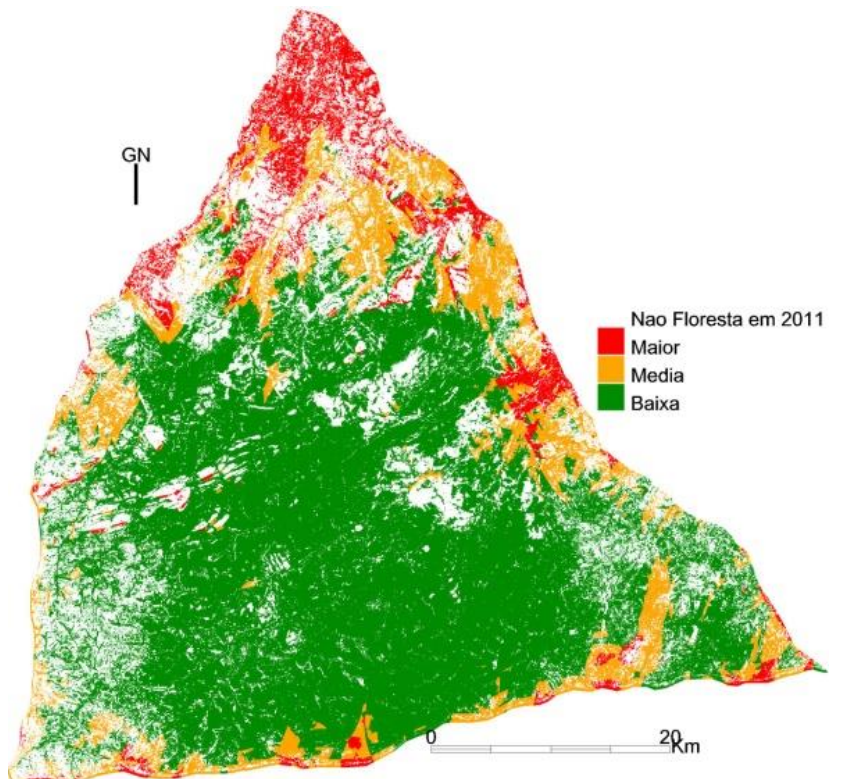

Fonte: Elaboração própria. 
A Figura 06 mostra o mapa da localização do desmatamento projetado para o período 2011 - 2020, produzido através do GEOMOD com base no desmatamento estimado para o ano 2020 (19485 ha), mapa de desmatamento de referência de 2011 e mapa de vulnerabilidade ao desmatamento.

\section{Figura 06: Mapa do desmatamento projectado para 2020.}

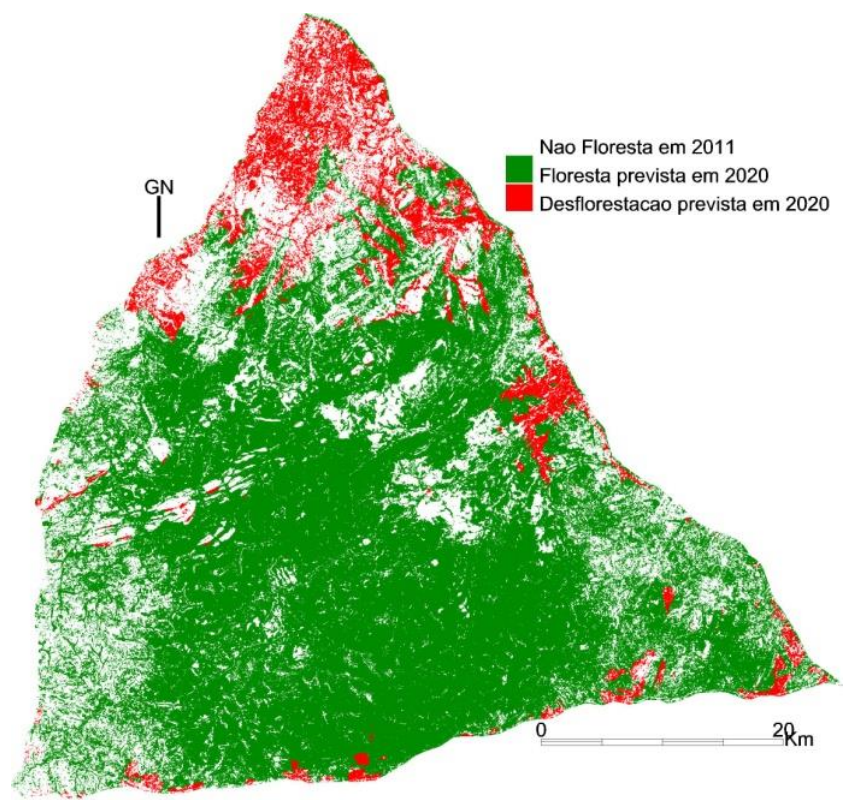

Fonte: Elaboração própria.

\section{DISCUSSÃO DOS RESULTADOS}

A abordagem aplicada para a determinação da taxa líquida de desmatamento assemelha-se, até certo ponto, à aplicada por Marzoli (2008) para estimar taxas de desmatamento em Moçambique entre 1990 - 2002. A taxa líquida obtida neste estudo $(0,72)$ situa-se ligeiramente acima da taxa média anual nacional $(0,58)$ mas, substancialmente inferior à taxa média anual na província de Nampula $(1,18)$. Refira-se, no entanto, que o sub período 2007/2011 apresenta uma taxa líquida $(1,10)$ próxima da média anual de Nampula. A taxa bruta é substancialmente superior à taxa líquida em todo período de referência, refletindo o fato de acumular o desmatamento registado em todos os anos em análise.

As taxas de desmatamento obtidas neste estudo sugerem que a reserva de Mecuburi se enquadra no quadrante III na matriz que divide as áreas candidatas a REDD de acordo com as taxas de desmatamento e a cobertura florestal. O quadrante III consiste de áreas com taxas de 
desmatamento maiores que 0,22\%/ano e cobertura florestal maior que 50\% (Da Fonseca et al., 2007).

Os mapas resultantes da modelagem mostram que a zona norte da reserva tem a maior área projetada para ser desmatada. Esta tendência segue o padrão da distribuição da densidade populacional que, por seu turno, encontra-se concentrada ao longo das estradas MecuburiMuite e Imala-Muite (Awasse \& Mushove, 2000; Aguiar Macedo,1968). O conhecimento da localização específica de áreas projetadas para o desmatamento permitirá estimar as emissões de $\mathrm{CO}_{2}$ correspondentes ao tipo de floresta e stock naquela localização, evitando-se o uso de valores médios conservadores. No caso particular da área de estudo, a maior área projetada paro desmatamento consiste basicamente de floresta aberta, a qual deverá conter stock de carbono relativamente baixo, comparado com a floresta densa mais concentrada na zona sul da reserva. Adicionalmente, pode ser importante para decidir sobre as estratégias e a melhor alocação de recursos que permita evitar ou, no mínimo, reduzir o desmatamento, tendo em conta as características sociais e económicas das comunidades locais.

Em várias outras regiões tropicais, autores destacam a importância da participação das comunidades locais na gestão dos recursos naturais, notadamente na conservação das florestas (Mondal \& Southworth, 2010; FAO, 2006). Na área de estudo, um programa de Maneio Comunitário dos Recursos Naturais (MCRN), iniciado em 1997 (FAO, 1996), envolveu algumas comunidades da reserva de Mecuburi através de comités locais de gestão de recursos naturais e grupos de interesse. Com este programa, a reserva passou a contar com fiscais comunitários de recursos naturais para além de se ter verificado um aumento da consciência ambiental no seio das comunidades (Sitoe \& Maússe - Sitoe, 2009). Nhantumbo \& Izidine (2009) reportaram que o MCRN teve um impacto positivo na conservação da biodiversidade e manutenção de serviços de ecossistema poucos anos após o seu início. No entanto, com o término do MCRN verificou-se uma total desintegração das estruturas locais de gestão dos recursos naturais o que, segundo Sitoe \& Maússe - Sitoe, 2009, provocou frustração no seio das comunidades locais, verificando-se envolvimento de elementos antes ativos na fiscalização de recursos naturais, em derrube da floresta para agricultura e outras finalidades.

Este trabalho constitui um ponto de partida para futuros estudos em torno de uma eventual intervenção do mecanismo REDD na reserva de Mecuburi. O sucesso dessa intervenção dependeria, em grande medida, de um envolvimento direto das comunidades locais e da capacidade de o REDD resultar em rendimentos superiores aos obtidos através de 
formas de uso de solo que conduzam ao desmatamento. De resto, desde 2007 que a produção de algodão, principal cultura de rendimento na área de estudo, enfrenta uma evolução negativa, o que pode favorecer o REDD. Segundo IAM, (2011), verifica-se um crescente desinteresse por parte dos produtores, como resultado de preços baixos de compra ao produtor, em virtude da instabilidade do mercado internacional.

\section{CONCLUSÃO}

A taxa bruta de desmatamento na Reserva de Mecuburi no período de 2002 a 2011 foi de 1,23\%, correspondendo a uma redução da área florestal de 2165,49 ha/ano. A taxa líquida situou-se nos $0,72 \%$ equivalente a redução da área florestal de 1231,86 ha/ano. Com a aplicação do Geomod foi possível mapear a localização de áreas propensas ao desmatamento para o período 2011 - 2020. Esses mapas podem servir de base para a definição de ações específicas para minimizar o desmatamento nas áreas identificadas.

\section{REFERÊNCIAS}

ACHARD, F., EVA, H., GLINNI, A., MAYAUX, P., RICHARDS, T., STIBIG, H. J. (EDS.). Identification of deforestation hot spot areas in the humid tropics. Trees Publication Series B, No. 4, 1998.

AGUiAR MACEDO, J. M. A Reserva Florestal de Mecubúri. Comunicação No 22. Lourenço Marques: Instituto Nacional de Investigação Agronómica de Moçambique, 1968.

ALLEN, J. C \& BARNES, D. F., The causes of deforestation in developing countries. Annals of the Association of American Geographers, 75 (2), p. 163-184, 1985.

ANGELSEN, A \& KAIMOWITZ, D., Rethinking the causes of deforestation: lessons from economic models. The World Bank Research Observer, 14, p. 73 - 98, 1999.

ARGOLA J.. Causas de mudança de cobertura florestal na região do Corredor da Beira. 2004. Tese (Licenciatura em Engenharia Florestal), Universidade Eduardo Mondlane, Maputo.

AWASSE, A \& MUSHOVE, P. Um modelo de Gestão de uma Reserva Florestal. FAO/MOZ-056, Internal Report, Mimeo, 2000.

BARROW, C. J. Land degradation: Development and breakdown in terrestrial environments. Cambridge: Cambridge University Press, 1991.

BROWN, S., ANDRASKO, K., HALL, M., DUSHKU, A., RUIZ, F., MARZOLI, W., MASERA, O., GUERRERO, G., DEJONG, B. Baselines for land-use change in the tropics: 
application to avoided deforestation projects. Mitigation Adaptation Strategy Global Change, 12, p. $10-26,2006$.

CAMPBELL, B. The Miombo in Transition: Woodlands and Welfare in Africa. Bogor, CIFOR, 1996.

CELENDER, N: Miombo Woodland in Africa- Distribution, Ecology and Pattern Versitet. International Rural Development Center. Arbest sapport, Working paper 16, Uppsala Suécia, 1983.

CINCOTTA, R. P \& ENGELMAN, R. Nature's place: Human population and the future of biological diversity. Population Action International. Washington, DC, 2000.

COSTA, F. Reservas Florestais de Nampula: Situação actual e Perspectivas. Maputo, Relatório de Consultoria, 1998.

DA FONSECA, G. A. B., RODRIGUEZ, C. M., MIDGLEY, G., BUSCH,J, HANNAH, L \& MITTERMEIER, R. A. No Forest Left Behind. PLoS Biol, 5(8), 2007.

DAILY, G. C., SO* DERQVIST, T., ANIYAR, S., ARROW, K., DASGUPTA, P., EHRLICH, P. R. The value of nature and nature of value. Science, 289, p. 395 - 396, 2000.

DEFRIES, R., FIELD, C., FUNG, I., COLLATZ, G., BOUNOUA, L. Combining satellite data and biogeochemical models to estimate global effects of human-induced land cover change on carbon emissions and primary productivity. Global Biogeochemical Cycles, 13, p.803-815, 1999.

FAO. Global Forest Resources Assessment 2005. Rome, Main Report, 2006.

FAO. Support for community Forestry and Wildlife Management. Maputo, Projecto GCP/MOZ/056/NET/Government Cooperative Programme, 1996.

FRA. Forest resources assessment 1990 -Tropical countries. Forestry paper, 112, 1993.

FROST, P. The Ecology of Miombo Woodlands. In: Campbell (Orgs). The Miombo in transition Woodland and Welfare in Africa. Bogor, CIFOR, 1996, p. 19 - 39.

GASTELlU-ETCHEGORRY, J.P., SINULINGGA, A.B. Designing a GIS for the study of forest evolution in Central Java. Tijdshcrift voor Economishe en Sociale Geografie, 79 (2), p. $93-103,1988$.

GEIST, H. J., \& LAMBIN, E. F. What drives tropical deforestation? A meta-analisys of proximate and underlying causes of deforestation based on subnational scale case study evidence. LUCC Report Series No. 4. University of Louvain, Louvainla - Neuve, 2001.

HALL, C. A. S., TIAN, H., QI Y., PONTIUS, G., CORNELL, J., UHLIG, J. Modeling spatial and temporal patterns of tropical land use change. J. Biogeogr, 22, p. 753 - 757, 1995.

HARRIS, N. L., PETROVA, S., STOLLE, F., BROWN, S. Identifying optimal areas for REDD intervention: East Kalimantan, Indonesia as a case study. Environmental Research Letter, 3 (2008) 035006, p. 1 - 11, 2008. 
HECHT, S. B. Environment, development, and politics:capital accumulation and the livestock sector in Amazonia. World Development, 13 (6), p. 663 - 684, 1985.

HOLBEN, B.N., KAUFAMAN,Y. J., KENDALL, J.D. NOAA-11AVHRRvisible and nearIR inflight calibration. International Journal of Remote Sensing, p. 1511 - 1519, 1990.

HOUGHTON, R. A., SKOLE, D. L., NOBRE, C. A., HACKLER, J. L., LAWRENCE, K. T., CHOMENTOWSKI, W. H. Annual fluxes of carbon from deforestation and regrowth in the Brazilian Amazon. Nature, 403, p. 301 - 304, 2000.

HUETTNER, M., LEEMANS, R., KOK, K \& EBELING, J. A comparison of baseline methodologies for Reducing Emissions from Deforestation and Degradation. Carbon Balance and Management, 4:4. 2009.

IAM. Relatório Sobre o Ponto de Situação do Subsector do Algodão. I Trimestre de 2011, Maputo, 2011.

IDRISI KLIMANJARO. Guide to GIS and Image Processing. Volume 2, USA, Idrisi Production - Clark Labs, p. 57 - 82, 2004.

JANSEN, L. J. M., BAGNOLI, M., CHIMENTI, S., DELL'AGNELLO, A., FOCACCI, M., MONACO, S. A., SORINI, P., STOPPIONI, S., URBANO, F. Satellite image interpretation of land-cover types in Manica and Maputo Provinces at nominal scale of 1:250,000 and at national level at nominal scale 1:1,000,000. Technical Report No. 1. Integrated Assessment of Mozambican Forests (AIFM) project. Agriconsulting S.p.A., Rome, 2006.

JANSEN, D. T., FREDEEN, A. L., \& WHEATE, R. D. Radiometric correction techniques and accuracy assessment for landsat TM data in remote forested regions. Canadian Journal of Remote Sensing, 32, p. 330 - 340, 2006.

MAE. Perfil de Desenvolvimento do Distrito de Mecuburi - Nampula. Ministério da Administração Estatal. Maputo, 2005.

MARZOLI, A. Avaliação Integrada das Florestas de Moçambique. Maputo, Agriconsulting, 2008.

MARTENS, B., SUNDERLIN, W., NDOYE, O., LAMBIN, E. F. Impact of macro-economic change on deforestation in South Cameroon: integration of household survey and remotelysensed data. World Development, 28, p. 983 - 999, 2000.

MATHER, A. S \& NEEDLE, C. L. The relationships of population and forest trends. The Geographical Journal 166 (1), p. 2 - 13, 2000.

MICOA. Estratégia Nacional de Redução de Emissões por Desmatamento e Degradação Florestal. Reduzir as emissões de carbono e a pobreza melhorando o maneio das florestas. Documento para debate, Maputo, 2010. 
MONDAL, P \& SOUTHWORTH, J. Protection vs. commercial management: Spatial and temporal analysis of land cover changes in the tropical forests of Central India. Forest Ecology and Management, 259 (2010), p. 1009 - 1017, 2010.

NHANTUMBO, I \& IZIDINE, S. Preparing for REDD in dryland forests: Investigating the options and potential synergy for REDD payments in the miombo eco-region (Mozambique country study). London, International Institute for Environment and Development (IIED), 2009.

NUALCHAWEE, K., MILLER, L., TOM, C., CHRISTENSON, J., WILLIAMS, D. Spatial inventory and modeling of shifting cultivation and forest land cover of northern Thailand with inputs from maps, air photos and Landsat. Texas, Texas A \& M University, College Station, 1981.

PERUMAL, K \& BHASKARAN, R. Supervised Classification Performance of Multispectral Images. Journal of Computing, Volume 2, Issue 2, 2010.

PONTIUS JR, R. G. Statistical methods to partition effects of quantity and location during comparison of categorical maps at multiple resolutions Photogrammetric Engineering \& Remote Sensing 68, p. 1041-1049, 2002.

PONTIUS JR, R. G., CORNELL, J \& HALL, C. A. S. Modeling the spatial pattern of landuse change with GEOMOD2: application and validation for Costa Rica. Agric. Ecosyst. Environ. 85, p. 191-203, 2001.

PONTIUS JR, R. G \& CHEN, H. Modeling With Geomod. Clark University, 2006.

PUYRAVAUD, J. P. Standardizing the calculation of the annual rate of deforestation. Forest Ecology Management. 177, 593 - 596, 2003.

RIBEIRO, N., MUSHOVE, P., AWASSE, A \& SIMANGO, S. Caracterização Ecológica da Floresta de Galeria do Rio Mecubúri na Reserva Florestal de Mecuburi, Província de Nampula. Maputo, UICN/UEM/DNFFB, 2002.

RICHARDS, J. A \& JIA, X. Remote Sensing Digital Image Analysis. An Introduction. 3th Edition, Springer, 1999.

RICHARDS, J. A. Remote Sensing Digital Image Analysis: An Introduction. SpringerVerlag, p. 265 - 290, 1995.

RODGERS, A., SALEHE, J \& HOWARD, G. The Ecology of Miombo Woodlands. Em B. B. Campbell (Orgs), The Miombo in transition: Woodlands and welfare in Africa. Bogor, CIFOR, 1996, p. $11-57$

ROSE, S., HELAL, A., EICKHOUT, B., FISHER, B., KUROSAWA, A., RAO, S., RIAHI, K. \& VAN VUUREN, D. Land in climate stabilization modeling: Initial observations. Energy Modeling Forum Report, Stanford University, 2007.

SAKET, M. Report on the updating of the exploratory national forest inventory. Maputo, FAO/UNDP MOZ/92/013 Project document, MADER/DNFFB/UIF, 1994 
SCARAMUZZA, P., MICIJEVIC, E \& CHANDER, G. SLC gap-filled products, Phase 1 Methodology. Disponível em: http://landsat.usgs.gov/documents/SLC_Gap_Fill_Methodology.pdf $\quad$ (visitado a 29 de Dezembro de 2011), 2004.

SEDANO, F., GONG, P., FERRÃO, M. Land-cover assessment with MODIS imagery in Southern African miombo ecosystems. Remote Sensing of Environment 98, p. 429 - 441, 2005.

SITOE, A. \& MAÚSSE - SITOE, S. Construindo parcerias florestais: potencial das reservas florestais na redução do desmatamento com participação das comunidades locais. Maputo. (s/d).

SKOLE, D \& TUCKER, C. Tropical deforestation and habitat fragmentation in the Amazon: Satellite data from 1978 to 1988. Science, 260, p. 1905 - 1909, 1993.

SWAIN, P. H., \& DAVIS, S. M. Remote sensing: The quantitative approach. New York' McGraw-Hill, 1978.

THE YALE CENTER FOR EARTH OBSERVATION. Filling Gaps in Landsat ETM Images, 2011.

TOKOLA, T., LÖFMAN, S., \& ERKKILÄ, A. Relative calibration of multitemporal Landsat data for forest cover change detection. Remote Sensing of Environment, 68, p. 1 - 11, 1998.

TUCKER, C. J., GRANT, D. M., DYKSTRA, J. D. NASA's Global Orthorectified Landsat Data Set. Photogrammetric Engineering \& Remote Sensing, 70 (3), p. 313 - 322, 2004.

UNFCCC. Background paper for the workshop on reducing emissions from deforestation in developing countries, August 2006, Rome, Italy. Part I. Scientific, socio-economic, technical and methodological issues related to deforestation in developing countries. Working paper No.1. 2006.

USGS. Imagery for Everyone: Timeline set to release entire USGS Landsat archive at no charge. Technical Announcement, 2008.

USGS. Shuttle Radar Topography Mission, 1 Arc Second scene SRTM_u03_n008e004, Unfilled Unfinished 2.0, Global Land Cover Facility, University of Maryland, College Park, Maryland, 2004.

Recebido em Abril de 2016

Aprovado em Junho de 2016

Publicado em Junho de 2016 